NISSUNA UMANA INVESTIGAZIONE SI PUO DIMANDARE VERA SCIENZIA S'ESSA NON PASSA PER LE MATEMATICHE DIMOSTRAZIONI LEONARDO DA VINCI

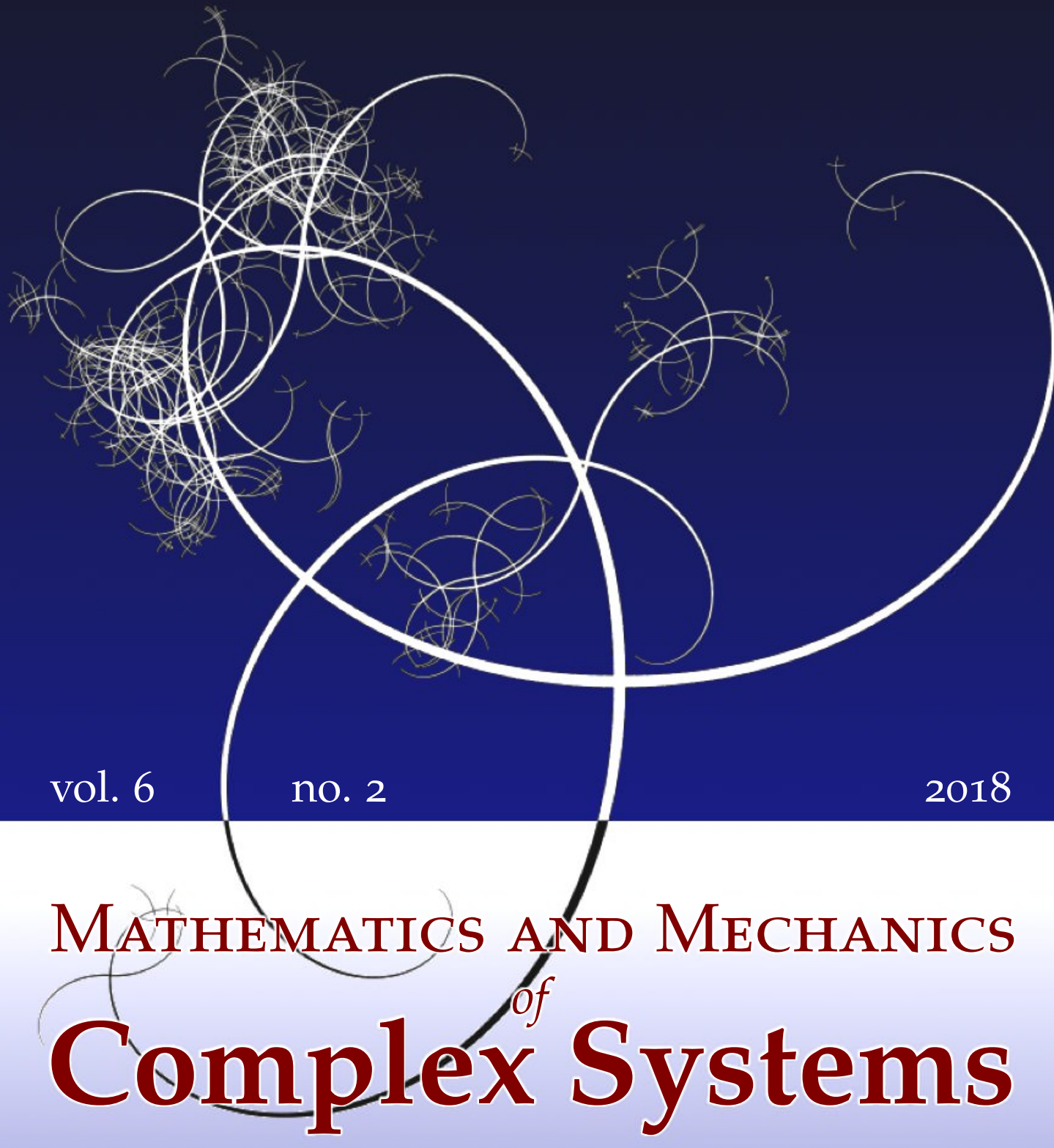

LuCA Placidi, Emilio BARChiesi AND ANIL MisRa

A STRAIN GRADIENT VARIATIONAL APPROACH TO DAMAGE: A COMPARISON WITH DAMAGE GRADIENT MODELS AND

NUMERICAL RESULTS 


\title{
A STRAIN GRADIENT VARIATIONAL APPROACH TO DAMAGE: A COMPARISON WITH DAMAGE GRADIENT MODELS AND NUMERICAL RESULTS
}

\author{
LuCA Placidi, EMIlio BARChiesi AND ANIL MisRa
}

The global response of experimental uniaxial tests cannot be homogeneous, because of the unavoidable presence of localized deformations, which is always preferential from an energetic viewpoint. Accordingly, one must introduce some characteristic lengths in order to penalize deformations that are too localized. This is what leads to the concept of nonlocal damage models. The nonlocal approach employs nonlocal terms in the internal deformation energy in order to control the size of the localization region. In phase-field models and, in general, in gradient models, dependence of the internal energy upon the first gradient of damage is assumed, while in our approach the nonlocality is given by the dependence of the internal energy upon the second gradient of the displacement field. A discussion of the advantages and challenges of using the gradient of damage and of using the second gradient of the displacement field will be addressed in the present paper. A variational inequality is formulated and partial differential equations (PDEs), boundary conditions (BCs), and Karush-KuhnTucker (KKT) conditions will be derived within the framework of 2D strain gradient damage mechanics. A novel dependence of the stiffness coefficients with respect to the damage field will also be discussed. Further, an explicit derivation of the damage field evolution in loading conditions will be provided. Finally, a numerical technique based on commercial software has been introduced and discussed for a couple of standard problems.

\section{Introduction}

1.1. A short overview. The literature on regularized damage laws, or so-called phase-field models, when the regularization is performed on the damage variable, has become quite intense in these last ten years. It has been proved, e.g., in [Lorentz and Andrieux 2003], that a regularization through the introduction of the gradient of damage allows one to overcome issues related to localization and mesh-dependency. Moreover, rigorous proofs of the convergence of such models

\section{Communicated by Jean-Jacques Marigo.}

MSC2010: 74C05, 74R99.

Keywords: strain gradient, damage mechanics, variational inequality, dissipation, regularization. 
towards the Griffith model of brittle fracture have strengthened such choice of regularization through the gradient of the damage variable; see for example [Ambrosio and Tortorelli 1990; Sicsic and Marigo 2013]. Thus, the nonlocality is customarily given by the dependence of the internal energy $U$ upon the first gradient of damage $\nabla \omega$ [Marigo 1989; Comi 1999; Pham et al. 2011a; Miehe et al. 2016]. A fully nonlocal model, in the Piola-peridynamic framework [dell'Isola et al. 2015a], has also been developed in the literature, like in, e.g., [Bažant and Jirásek 2002; Bažant and Pijaudier-Cabot 1988]. Another interesting and effective approach is due to [Forest 2009]. In the approach presented in this paper the nonlocality is given by the dependence of the internal energy upon the second gradient of the displacement field. In other words, the internal elastic energy per unit volume $U$ is assumed to be a function not only of the strain $G$, but also of its gradient $\nabla G$. This approach is not new in damage continuum mechanics; see also [Peerlings et al. 2001; Mühlich et al. 2013; Zybell et al. 2009; Oliver-Leblond et al. 2016]. Beyond the convergence of some damage gradient models towards the Griffith model for brittle fracture, the main advantage of using the gradient of damage is simply due to the fact that handling the gradient of a scalar (i.e., the damage field) is easier than dealing with the gradient of a tensor (i.e., the strain). A first consequence of this fact is that the number of constitutive parameters for a damage gradient model is lower than that of a strain gradient model, and this will be discussed in more detail in the next subsection. In the remainder of this section we investigate and present two main advantages of using the strain gradient approach. First of all, an interpretation of those boundary conditions that are necessary to ensure the uniqueness of the solution is guaranteed only for strain gradient models and not for damage gradient models. This issue is very important when performing experimental and numerical parameter identification. Secondly, regularization in the elastic phase is achieved only with strain gradient models and not with damage gradient models.

1.2. Number of constitutive parameters. Let us consider for simplicity the isotropic case. For the strain gradient model the number of constitutive coefficients to be identified (in addition to the standard Lamé coefficients) is 5 for the 3D case and 4 for the 2D case. For the damage gradient model, in addition to the standard Lamé coefficients, we have 1 further parameter. In order to identify the constitutive parameters, an experimental procedure is necessary. In this regard, many attempts [Placidi et al. 2015; 2017; Rahali et al. 2016] have been exploited in the framework of strain gradient elasticity.

1.3. Interpretation of boundary conditions. In continuum damage mechanics, the kinematics (see also Figure 1) is defined by both the displacement $u(X, t)$ (or the placement $\chi(X, t))$ and the damage $\omega(X, t)$ fields. In the damage gradient approach one assumes always natural boundary conditions, as is shown in Figure 2, 


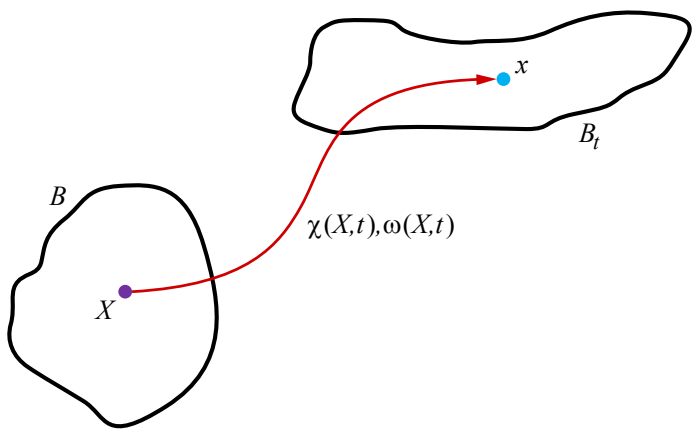

Figure 1. Kinematics in continuum damage mechanics.

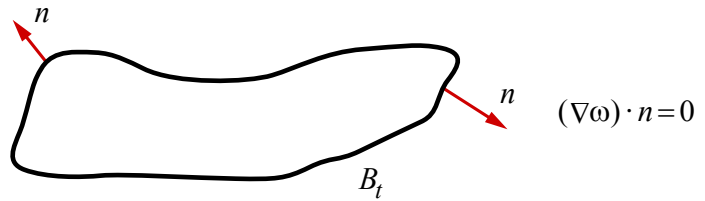

Figure 2. Natural boundary conditions for the damage gradient approach.

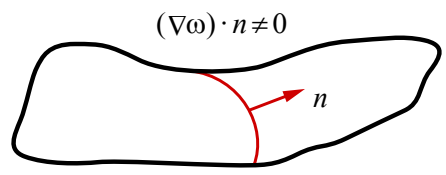

Figure 3. An internal boundary (a line in the present $2 \mathrm{D}$ case) is depicted, where $n$ is one of its unit normals. The internal boundary is chosen in such a way that the projection of the damage gradient on the unit normal $n$ is nonzero.

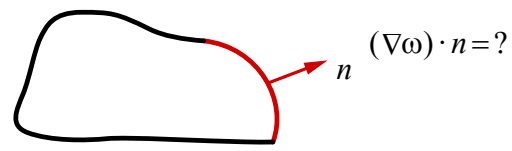

Figure 4. External boundary conditions for the damage gradient approach that guarantee the same solution of the boundary value problem represented in Figure 2.

where $n$ is the external unit normal. With these natural boundary conditions, which are represented in Figure 2, one obtains a solution in terms of the damage field $\omega(X, t)$ such that there exists an internal boundary where

$$
(\nabla \omega) \cdot n \neq 0
$$




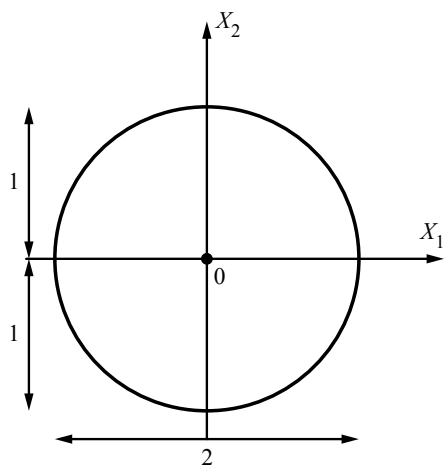

Figure 5. Domain of the elastic problem defined in (3).

Such an internal boundary is depicted, e.g., in Figure 3. Let us now perform a so-called Cauchy-cut over the internal boundary represented in Figure 3 and take the left part in such a way that the unit normal $n$ defines its external unit normal. We now take into account the boundary value problem, as in Figure 4, such that the solutions of the problems represented in Figures 3 and 4 are the same if restricted over the domain of Figure 4. Which kind of boundary conditions should we assume for the damage gradient? If we assume zero natural boundary conditions, then the solutions of the problems in Figures 3 and 4, restricted over the domain of Figure 4, are different. Because of the uniqueness of the solution, in order for the problems in Figures 3 and 4 to have the same solution, we should have nonzero natural boundary conditions. Thus, in the damage gradient approach one faces the problem of interpreting the natural boundary conditions. It is worth noting that the interpretation of the extra boundary conditions in the strain gradient approach, in terms of the normal gradient of displacement and/or double force and in terms of vertex-contact actions, is standard for elastic strain gradient models.

1.4. Regularization of the elastic phase. In order to support the claim that a regularized scheme is necessary also for the elastic phase, we consider the following example (personal communication of Pierre Seppecher). The number of examples of this kind is very large. However, what we show here is simple to conceive and deserves a short illustration. Let us find, among all the displacement fields that satisfy the boundary conditions

$$
u / u(O)=\hat{e}_{3}, \quad u(\partial \Omega)=0,
$$

the solution to the second-gradient elastic problem

$$
\inf \int_{\Omega}\|\nabla \nabla \boldsymbol{u}\|^{2},
$$


where $\Omega \subseteq \mathbb{R}^{2}$ is the circle of radius equal to 1 in Figure 5 and $\hat{e}_{3}$ is the out-of-plane unit vector. It is possible to prove that the solution to (3) is

$$
u=\frac{2}{3} r^{2} \ln r-r^{2}+1
$$

and that the infimum, which is in fact a minimum, is

$$
\int_{\Omega}\|\nabla \nabla \boldsymbol{u}\|^{2}=\pi \frac{16}{3} \text {. }
$$

This means that the energy attained in correspondence of the solution is finite, as one would expect. If the energy to be minimized, among all the displacement fields satisfying the (2), is of first-gradient type, e.g., it is given by

$$
\inf \int_{\Omega}\|\nabla \boldsymbol{u}\|^{2}
$$

an explicit analytical solution can still be found and reads as

$$
u=\lim _{\varepsilon \rightarrow 0} \frac{\ln r}{\ln \varepsilon} .
$$

In this case, the infimum is

$$
\int_{\Omega}\|\nabla \boldsymbol{u}\|^{2}=-\lim _{\varepsilon \rightarrow 0} \frac{2 \pi}{\ln \varepsilon}=0,
$$

which means that the energy attained in correspondence of the solution is zero, a fact that is clearly not reasonable on a physical ground.

\section{The variational inequality and the derivation of governing equations}

In order to formulate governing equations for nonstandard models, it is useful to use a variational procedure. The reason for such a choice is that the definition of those boundary conditions that guarantee uniqueness of the solution is straightforward in this way. A variational principle of maximum plastic work has been derived already by Hill [1948]. Further contributions are due to, among others, [Maier 1970; Bažant 1980; Bourdin et al. 2008; Pham et al. 2011b; Marigo 1989; Amor et al. 2009; Pham and Marigo 2010a; 2010b; Reddy 2011a; 2011b].

2.1. Kinematics of the model. As shown in Figure 1, the kinematics of the model is given by the displacement field $u$, which is an observable state variable

$$
u:\left(\mathbb{R}^{2} \supseteq \mathscr{B},[0, T]\right) \rightarrow \mathbb{R}^{2},
$$

and by the damage field $\omega$, which is an internal state variable

$$
\omega:\left(\mathbb{R}^{2} \supseteq \mathscr{B},[0, T]\right) \rightarrow[0,1],
$$


with $\omega=0$ corresponding to the undamaged material and $\omega=1$ to the failure state. Further, we don't take into account any healing mechanism, and this introduces the unilateral (entropic) constraint

$$
\dot{\omega} \geq 0
$$

2.2. The variational inequality. We assume a total deformation energy functional $\mathscr{E}(u, \omega)$ of the kind which has been discussed so far (i.e., including strain gradient). Thus, we evaluate its variation $\delta \mathscr{E}(u, \omega, \delta u, \delta \omega)$. Finally, the variational inequality

$$
\delta \mathscr{E}(u, \omega, \dot{u}, \dot{\omega}) \leq \delta \mathscr{E}(u, \omega, v, \beta) \quad \text { for all } v \text { and for all } \beta \geq 0
$$

is assumed for any admissible virtual velocity fields $\beta$ and $v$. As remarked in [Marigo 1989], inequality (12) states that the actual energy release rate is not smaller than any possible one. Thus, it constitutes a kind of principle of maximum energy release rate.

2.3. The total energy functional in the strain gradient damage $2 D$ case. The total energy functional which is here investigated is defined as

$$
\begin{aligned}
\mathscr{E}(u, \omega)=\int_{\mathscr{B}}\left[U(G, \nabla G, \omega)-b^{\mathrm{ext}} \cdot u-m^{\mathrm{ext}} \cdot \nabla u\right] d A \\
\quad-\int_{\partial \mathscr{B}}\left[t^{\mathrm{ext}} \cdot u+\tau^{\mathrm{ext}} \cdot[(\nabla u) n]\right] d s-\int_{[\partial \partial \mathscr{B}]} f^{\mathrm{ext}} \cdot u,
\end{aligned}
$$

where a standard second-gradient elastic energy, with $G$ the symmetric part of the displacement gradient, has been complemented with an isotropic local damage dissipation term. The $2 \mathrm{D}$ isotropic quadratic internal deformation energy density functional accounting for damage is

$$
U(G, \nabla G, \omega)=U_{e}(G, \nabla G, \omega)+\frac{k}{2} \omega^{2},
$$

where $k$ is the resistance to damage. The elastic part $U_{e}(G, \nabla G, \omega)$ of the internal energy that is here considered is

$$
\begin{aligned}
U_{e}(G, \nabla G, \omega)=2 \mu G_{12}^{2}+\frac{1}{2} \lambda\left(G_{11}+G_{22}\right)^{2}+\mu\left(G_{11}^{2}+G_{22}^{2}\right) \\
+\frac{B}{2}\left(G_{11,1}{ }^{2}+G_{22,2}{ }^{2}\right)+2 A\left(G_{12,1}{ }^{2}+G_{12,2}{ }^{2}\right) \\
+\left(\frac{3 A}{2}-B+C+2 D\right)\left(G_{11,2}^{2}+G_{22,1}{ }^{2}\right) \\
+(A+B-2 C)\left(G_{11,1} G_{12,2}+G_{12,1} G_{22,2}\right) \\
+(-4 A+2 B-4 D)\left(G_{12,2} G_{22,1}+G_{11,2} G_{12,1}\right) \\
+\left(-\frac{A}{2}-\frac{B}{2}+C+2 D\right)\left(G_{11,2} G_{22,2}+G_{11,1} G_{22,1}\right),
\end{aligned}
$$


where the stiffness coefficients $\lambda, \mu, A, B, C$, and $D$ all depend upon damage $\omega$. The dependence of the elastic coefficients upon damage is illustrated in the next subsection.

2.4. Dependence of the elastic coefficients upon damage. In our model Lamé constants are, as it is customarily assumed in damage mechanics, a decreasing function of damage,

$$
\lambda=\lambda_{0}(1-\omega), \quad \mu=\mu_{0}(1-\omega),
$$

while second-gradient elastic stiffnesses are increasing with increasing damage,

$$
A=A_{0}(1+n \omega), \quad B=B_{0}(1+n \omega), \quad C=C_{0}(1+n \omega), \quad D=D_{0}(1+n \omega), \quad n \in \mathbb{R} .
$$

The reason for such a choice is that the state of damage is by itself a kind of measure of the microstructures of the continuum. When modeling some classes of phenomena (e.g., the behavior of laminate composites, where damage is spreading without localizing too much), it is reasonable to conceive a model in which the postulated dependencies (17) of $A, B, C$, and $D$ upon damage are appropriate. For further details the reader is referred to the complete formulation in [Placidi 2015; 2016]. Moreover, in Section 4 some numerical results show the sensitivity of a certain solution upon the parameter $n$. Finally, it is worth noting that, in order to study the fracture propagation, equations (17) should change. However, this will be the topic of another work.

2.5. Derivation of governing partial differential equations. It is possible to prove that the variational inequality (12) reduces to the usual balance of momenta when arbitrary variations $\delta u$ and no variations $\delta \omega$, i.e., $\delta \omega=0$, are considered:

$$
\delta \mathscr{E}(u, \omega, \delta u, \delta \omega=0)=0 .
$$

By applying the localization theorem we get the system of PDEs

$$
\left(S_{i j}-T_{i j h, h}\right)_{, j}+b_{i}^{\text {ext }}-m_{i j, j}^{\text {ext }}=0 \quad \text { for all } X \in \mathscr{B},
$$

where stress and hyperstress are defined as

$$
S_{i j}=\frac{\partial U}{\partial G_{i j}}, \quad T_{i j h}=\frac{\partial U}{\partial G_{i j, h}} .
$$

2.6. Derivation of boundary conditions. For those points of $\partial \mathscr{B} \backslash[\partial \partial \mathscr{B}]$ where kinematical constraints on $u$ are not given, i.e., where $\delta u \neq 0$, we have the natural boundary conditions $t-t^{\mathrm{ext}}-m^{\mathrm{ext}} n=0$. For those points of the $\partial \mathscr{B} \backslash[\partial \partial \mathscr{B}]$ where kinematical constraints on $\nabla u n$ are not given, i.e., where $\delta \nabla u n \neq 0$, we have the

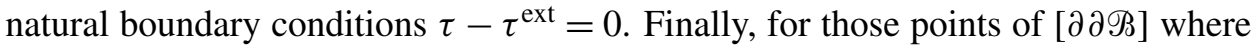


kinematical constraints on $u$ are not given, i.e., where $\delta u \neq 0$, we have the natural boundary conditions $f-f^{\text {ext }}=0$. For further details, i.e., for proper definitions of the contact actions $t, \tau$, and $f$, the reader is referred to the complete formulation in [Placidi et al. 2015; Placidi and El Dhaba 2017].

2.7. Derivation of the Karush-Kuhn-Tucker conditions. By choosing $v=\dot{u}$ and $\beta=0$ (followed by the choice $\beta=2 \dot{\omega}$ and then by the choice $\beta=\dot{\omega}$ ) in the variational inequality (12), it is possible to prove, by localization arguments, that $\frac{\partial U}{\partial \omega}$ and/or $\dot{\omega}$ (which is always nonnegative, i.e., $\dot{\omega} \geq 0$ ) must vanish for each point $X$ of $\mathscr{B}$ and time $t$

$$
\frac{\partial U}{\partial \omega} \dot{\omega}=0 \quad \text { for all } X \in \mathscr{B} \text {. }
$$

Thus, we are able to derive the so-called Karush-Kuhn-Tucker (KKT) conditions for damage mechanics simply from the variational principle (12).

2.8. Comments. For a fixed $\omega$, the behavior is (linear and) elastic. However, since $\omega$ evolves (quasistatically), the global behavior is inelastic and the effective Young's modulus is proportional to $(1-\omega)$. This corresponds to a global softening behavior. Further, given the choice of the dissipation energy (i.e., quadratic dependence upon damage), damage will increase from the very beginning and no purely elastic behavior is observed as if, e.g., a linear dependence upon damage was assumed. It is crucial, even if redundant, to remark that our model accounts for the fact that localization of strain and damage consists of a two-way interaction: localization of strain implies localization of damage and vice versa. Anyway, contrarily to what is done usually in damage mechanics, accounting for nonlocal behavior is not encoded in the (local) damage term (indeed this is not a phase field model, i.e., nonlocal/gradient damage). Accounting for nonlocal behavior is encoded in the dependence of the strain energy upon the strain gradient (advantages and challenges of this approach were explained in the previous section). It is possible to show that, without nonlocal terms, concentration of stress (strain) leads to a burst of damage (up to 1) in these very localized regions. Consequently, the first-gradient model works only for moderate levels of mean damage, being unable to capture, for instance, the softening process. Further, since strain gradient terms make us "pay" for the localization of strain (stress), they play the role of "limiters" against brutal failure. Thus, the model works up to higher levels of mean damage, being able to capture the softening process.

\section{Solution algorithm in incremental form}

Since $\dot{\omega} \geq 0$, KKT conditions (21) imply that

$$
\frac{\partial U}{\partial \omega}=0 \quad \vee \quad \dot{\omega}=0 \quad \text { for all } X \in \mathscr{B} .
$$


Then, we define a damage threshold $\bar{\omega}(G, \nabla G)$ such that

$$
\frac{\partial U}{\partial \omega}(G, \nabla G, \bar{\omega}(G, \nabla G))=0 .
$$

With the prescriptions (15) on the functional dependence of the elastic internal energy upon the strain and the strain gradient tensors, (14) on the functional dependence of the dissipation energy upon the entropic damage variable, and (16)-(17) on the functional dependence of the first- and second-gradient elastic stiffnesses upon the entropic damage variable, we obtain the form for the damage threshold

$$
\begin{aligned}
\bar{\omega}(X, t) & =\frac{\lambda_{0}+2 \mu_{0}}{k}\left(u_{1,1}^{2}+u_{2,2}^{2}\right)+2 \frac{\mu_{0}}{k} u_{1,2} u_{2,1}+\frac{\mu_{0}}{k}\left(u_{1,2}^{2}+u_{1,1}^{2}\right)+2 \frac{\lambda_{0}}{k} u_{1,1} u_{2,2} \\
- & n \frac{A_{0}}{k}\left(u_{1,22}^{2}+u_{2,11}^{2}\right)-n \frac{B_{0}}{k}\left(u_{1,11}^{2}+u_{2,22}^{2}\right)-n \frac{C_{0}}{k}\left(u_{1,12}^{2}+u_{2,12}^{2}\right) \\
- & 2 n \frac{D_{0}}{k}\left(u_{1,11} u_{2,12}+u_{2,22} u_{1,12}\right)-n \frac{B_{0}-C_{0}+A_{0}}{k}\left(u_{1,11} u_{1,22}+u_{2,11} u_{2,22}\right) \\
- & 2 n \frac{B_{0}-A_{0}-D_{0}}{k}\left(u_{1,12} u_{2,11}+u_{1,22} u_{2,12}\right) .
\end{aligned}
$$

First of all, an initial condition for both displacement and damage is assigned as $u(X, 0)=u_{0}(X)=0 \quad$ for all $X \in \mathscr{B}, \quad \omega(X, 0)=\omega_{0}=0 \quad$ for all $X \in \mathscr{B}$.

Once the initial condition is assigned, the displacement field $u_{i}(X)$ for the $i$-th step (with $i \in \mathbb{N}$ ) is derived from (18) as

$$
u_{i}=\arg \min _{u: \mathscr{乃} \rightarrow \mathbb{R}^{2}} \mathscr{E}\left(u, \omega_{i-1}\right),
$$

and the damage field $\omega_{i}(X)$ for the $i$-th step is derived from (21) as

$$
\omega_{i}=\max \left(\bar{\omega}\left(G_{i}, \nabla G_{i}\right), \omega_{i-1}\right),
$$

where here we intend $u_{i}$ and $\omega_{i}$ to be the values, at a certain point, of the displacement $u$ and damage $\omega$ at the time step $t_{i}$. It is worth noting that an a priori discretization of the time variable, that in the present quasistatic case is interpreted as an order parameter, must be performed.

The incremental formulation has been implemented in MATLAB. For simplicity, we performed only displacement-controlled numerical experiments and, at each step, the minimization problem in (26) is approximated by means of the weak form package of the FEM software COMSOL Multiphysics. The mesh is triangular, and it is Delaunay-tessellated (maximum element size $3.0 \times 10^{-4} \mathrm{~m}$ and minimum element size $\left.6.0 \times 10^{-7} \mathrm{~m}\right)$. When strain gradient constitutive coefficients $A=$ $B=C=D=0$ are null, quadratic Lagrangian shape functions are employed while, when they are greater than zero, cubic Hermite shape functions are used. The Newton-Raphson method is used to numerically solve the algebraic system 


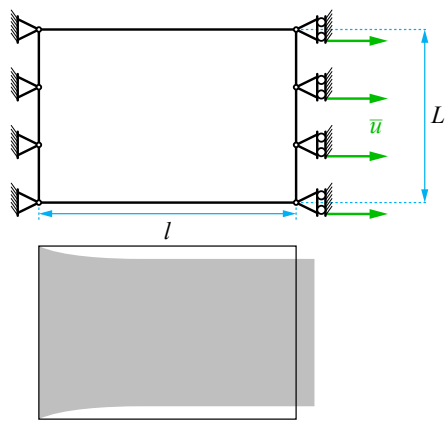

Figure 6. Tensile test (plain plate): reference domain with a schematic of BCs (top); vertical displacement of the deformed configuration for a linear elastic isotropic homogeneous body (bottom) is emphasized more than the horizontal displacement.

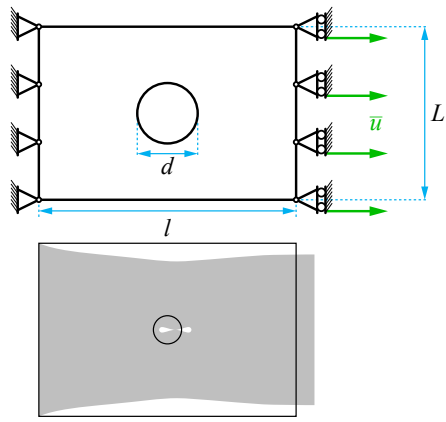

Figure 7. Tensile test (perforated plate): reference domain with a schematic of BCs (top); vertical displacement of the deformed configuration for a linear elastic isotropic homogeneous body (bottom) is emphasized more than the horizontal displacement.

coming from the Galerkin approximation. The computational time for each step is approximately $40 \mathrm{~s}$ with an Intel Core i7-6700HQ CPU at $2.60 \mathrm{GHz}$ and $16 \mathrm{~GB}$ RAM machine.

\section{Tensile tests of plain and perforated plate}

We investigate two simple geometries: a plain rectangle, like the one in Figure 6, and a perforated rectangle, like the one in Figure 7.

Numerical simulations have been performed with the constitutive coefficients illustrated in Table 1, where the Lamé coefficients $\lambda$ and $\mu$ depend in the standard way upon the Young's modulus and Poisson's ratio given in Table 1. Further, $L$ 


\begin{tabular}{|ccccc|}
\hline $\begin{array}{c}\text { Young's modulus }(Y) \\
75 \mathrm{GPa}\end{array}$ & $\begin{array}{c}\text { Poisson's ratio } \\
0.11\end{array}$ & $\begin{array}{c}k \\
75 \mathrm{kPa}\end{array}$ & $\begin{array}{c}L \\
20 \mathrm{~mm}\end{array}$ & $\begin{array}{c}l \\
30 \mathrm{~mm}\end{array}$ \\
\hline$\alpha_{1}^{0}, \alpha_{2}^{0}, \alpha_{3}^{0}, \alpha_{4}^{0}$ & $\alpha_{5}^{0}$ & $n$ & $d$ & \\
$m \cdot Y \cdot 1 \mathrm{~mm}^{2}$ & $\alpha_{1}^{0} / 2$ & 0 or 1 & $0.33 \mathrm{~mm}$ & \\
\hline
\end{tabular}

Table 1. Numerical values which are used in simulations.

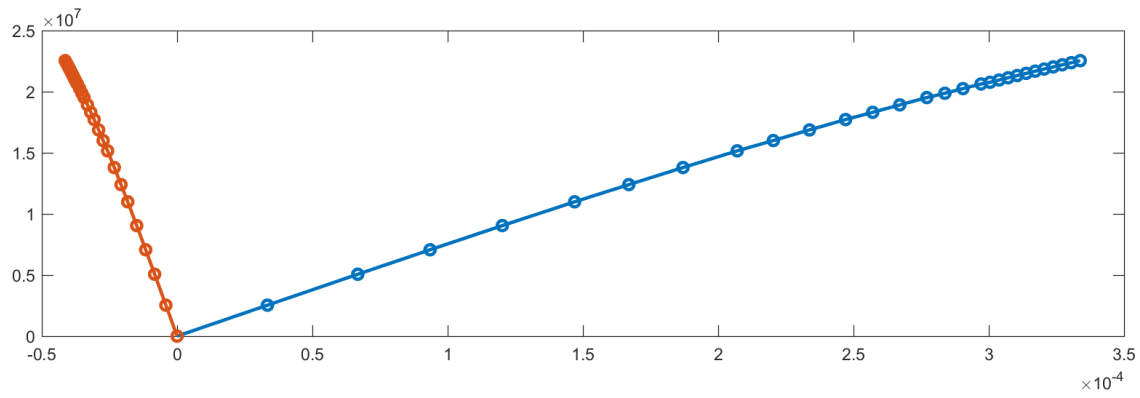

Figure 8. Tensile test (plain plate): $m=0 . \quad S_{11}(\mathrm{~Pa})$ (right side midpoint) versus $G_{11}$ (right side midpoint) (blue); $S_{11}(\mathrm{~Pa})$ (right side midpoint) versus $G_{22}$ (right side midpoint) (orange); $l=30 \mathrm{~mm} ; m=0 ; n=1$.

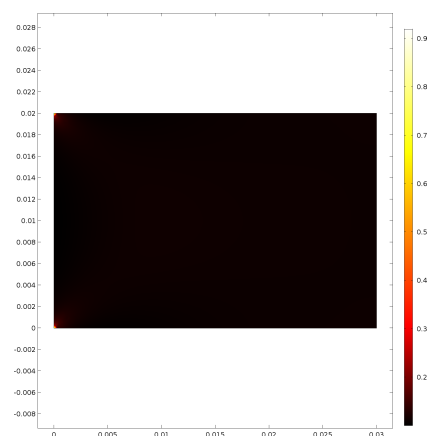

Figure 9. Tensile test (plain plate): $m=0$. Color map of $\omega$ at failure point; $l=30 \mathrm{~mm} ; m=0 ; n=1$. No boundary layer. Burst of damage in very localized zones.

and $l$ are the lengths of the sides of the rectangle and the undamaged secondgradient stiffnesses $A_{0}, B_{0}, C_{0}$, and $D_{0}$ are related to the 5 Mindlin's 3D coefficients illustrated in Table 1 as

$$
\left(\begin{array}{l}
A_{0} \\
B_{0} \\
C_{0} \\
D_{0}
\end{array}\right)=\left(\begin{array}{lllll}
0 & 0 & 2 & 2 & 2 \\
8 & 2 & 8 & 4 & 8 \\
2 & 1 & 1 & 3 & 5 \\
3 & 1 & 2 & 0 & 0
\end{array}\right)\left(\begin{array}{l}
\alpha_{1}^{0} \\
\alpha_{2}^{0} \\
\alpha_{3}^{0} \\
\alpha_{4}^{0} \\
\alpha_{5}^{0}
\end{array}\right) .
$$




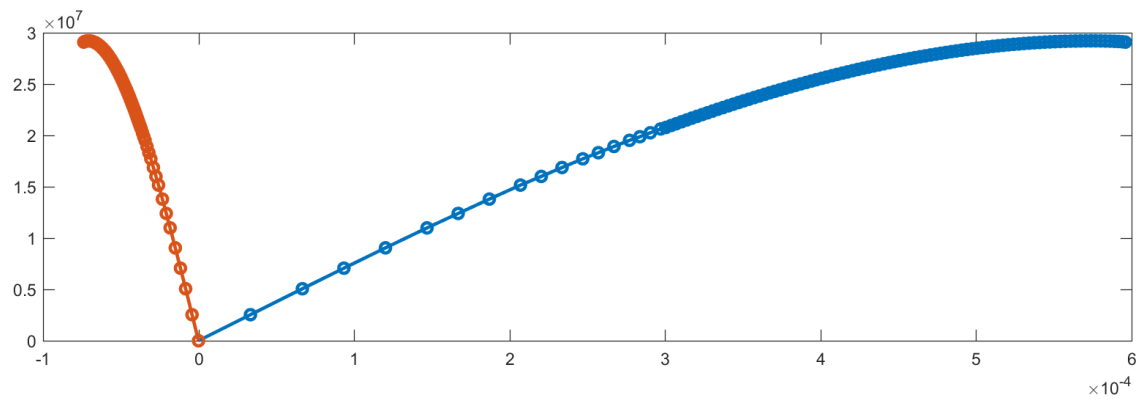

Figure 10. Tensile test (plain plate): $m=0.05 . S_{11}(\mathrm{~Pa})$ versus $G_{11}$ (blue); $S_{11}\left(\mathrm{~Pa}\right.$ ) versus $G_{22}$ (orange); $l=30 \mathrm{~mm} ; m=0.05$; $n=1$. This graphic shows strain-gradient-enabled softening.

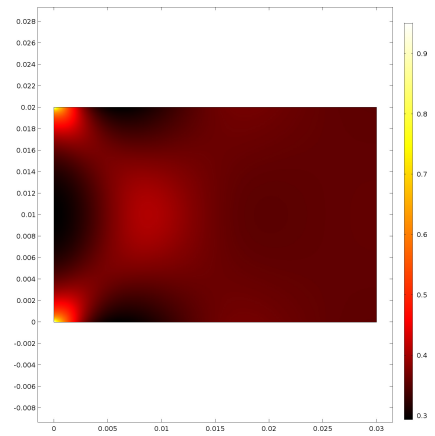

Figure 11. Tensile test (plain plate): $m=0.05$. Color map of $\omega$ at failure point; $l=30 \mathrm{~mm} ; m=0.05 ; n=1$.

Moreover, as is shown in Table $1, m$ is the (nondimensional) weight of secondgradient terms in the internal strain energy. Further, the parameter $n$, introduced in the constitutive assumptions (17), determines the effect of damage on the microstructure; i.e., damage can either affinely magnify $(n>0)$ or affinely shrink $(n<0)$ the square of the characteristic length that is, in millimeters, given by $\sqrt{m}$. Finally, $d$ is the diameter of the circular hole appearing in Figure 7. We make clear that the abscissa of the stress-strain plots which will be shown in the sequel is not equal to the ratio of the imposed displacement $\bar{u}$ to $l$ (i.e., it is not the global strain), but it is in fact the value of $G_{11}$ at the midpoint of the right side of the rectangular domain, i.e., the local strain.

In Figure 8, the stress-strain relationship for the tensile simulation of a plain plate is shown for a first gradient continuum, which means for $m=0$. A slight loss of material stiffness is observed, which however does not lead to any decrease in stress as strain increases. In Figure 9, a contour plot of the damage variable is shown at failure point, in the same example. We remark that the absence of 

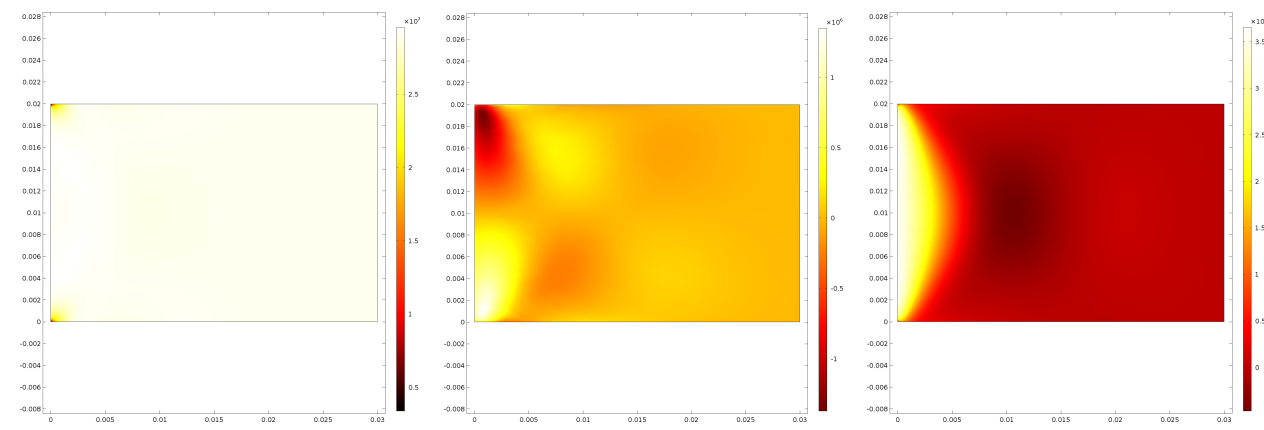

Figure 12. Tensile test (plain plate): $m=0.05$. Color map of $S_{11}(\mathrm{~Pa})$ (left), $S_{12}(\mathrm{~Pa})$ (center), $S_{22}(\mathrm{~Pa})$ (right) at failure point; $l=30 \mathrm{~mm} ; m=0.05 ; n=1$.

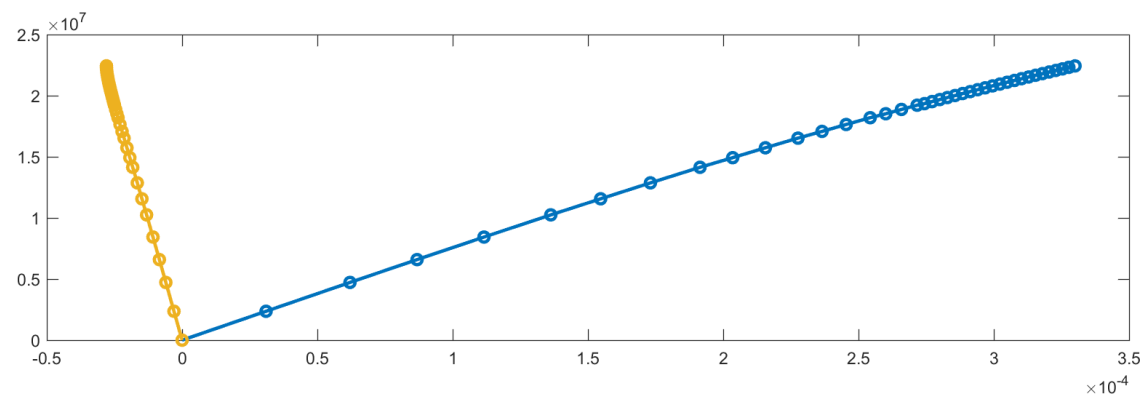

Figure 13. Tensile test (perforated plate): $m=0.05 . S_{11}(\mathrm{~Pa})$ versus $G_{11}$ (blue); $S_{11}(\mathrm{~Pa})$ versus $G_{22}$ (orange); $l=30 \mathrm{~mm} ; m=0.05$; $n=1$. This graphic does not exhibit softening. The value of $m$ required to have softening is relatively much higher.

nonlocal contributions to the internal strain energy density, which would prevent strain localization, results in the absence of any boundary layer and, thus, in a burst of damage in very localized zones. This fact leads to mesh dependency as, whatever the finite element characteristics (size and geometry of mesh and shape function), the first failure is always observed in one single finite element. In Figure 10, which represents the stress-strain diagram for the experiment in Figure 6 and for $m=0.05$, a loss of material stiffness is observed as well. Since the increase of damage due to localization is slowed down by the presence of second-gradient contributions, failure of the material occurs well after that in the case of $m=0$ and a stationary point of the stress-strain relationship is observed for a longitudinal strain corresponding to approximately $G_{11}=5.8 \times 10^{-4}$. In Figure 11, a contour plot of the damage variable is shown at failure point for the same example. We remark that the presence of nonlocal contributions to the internal strain energy density 


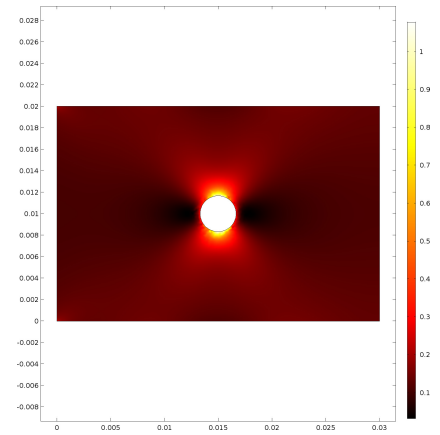

Figure 14. Tensile test (perforated plate): $m=0.05$. Color map of $\omega$ at failure point; $l=30 \mathrm{~mm} ; m=0.05 ; n=1$. This graphic is coherent with well known analytical results in the theory of firstgradient linear homogeneous isotropic elasticity.
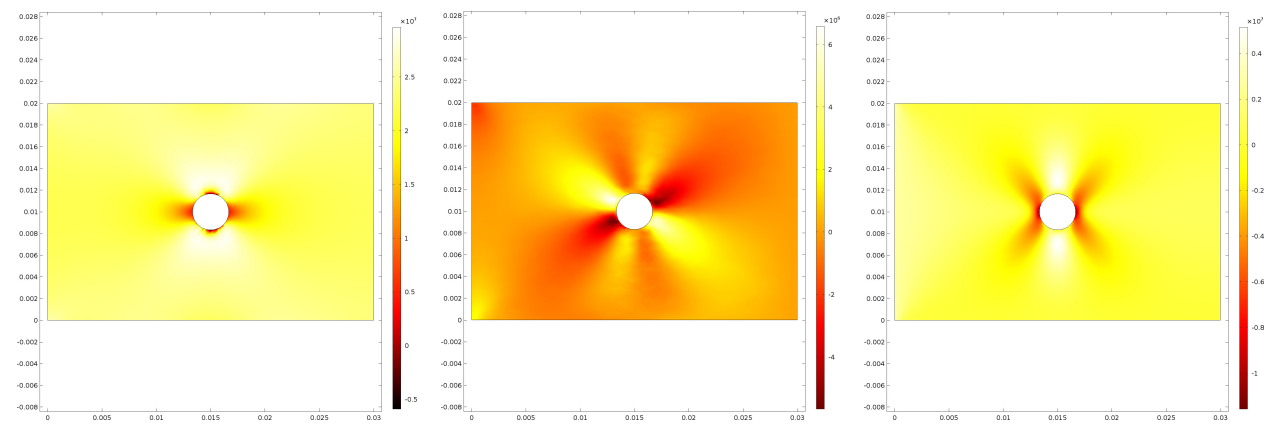

Figure 15. Tensile test (perforated plate): $m=0.05$. Color map of $S_{11}(\mathrm{~Pa})$ (left), $S_{12}(\mathrm{~Pa})$ (center), $S_{22}(\mathrm{~Pa})$ (right) at failure point; $l=30 \mathrm{~mm} ; m=0.05 ; n=1$.

prevents strain localization and mesh dependency is not observed since the regions with the highest value of damage at the left corners of the specimen are larger than the size of a single finite element. In Figure 12 the contour plots at failure point of the components of the stress tensor are shown. Figures 13, 14, and 15 show, respectively, the stress-strain curve, the damage contour plot at failure point, and the contour plots of the components of the stress tensor for the test described in Figure 7, when $m=0.05$. As is clear from Figure 14, failure occurs at the intersection of the transversal axis of the rectangular specimen with the perimeter of the internal circular void and mesh dependency is again avoided by the presence of second-gradient contributions. Still, the characteristic length introduced by means of second gradient is not sufficiently large to limit the increase of damage up to failure and, thus, to see a change of sign in the derivative of the stress-strain 


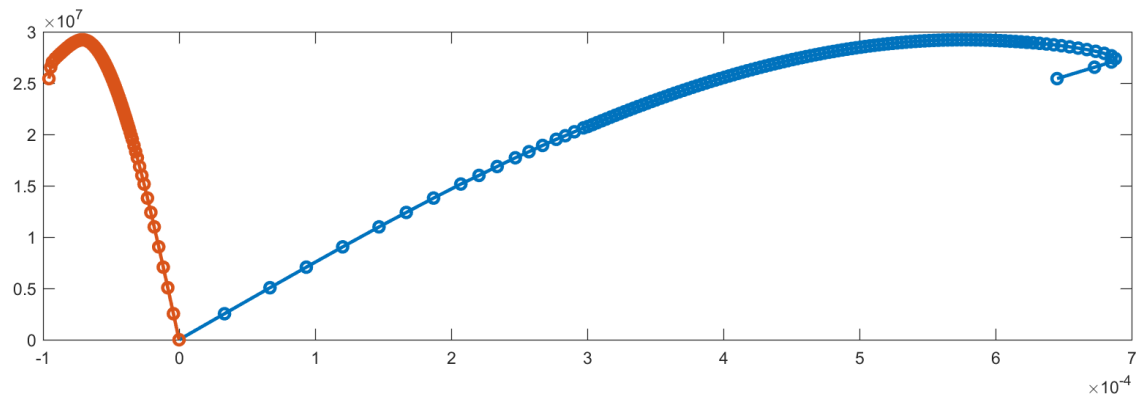

Figure 16. Tensile test (plain plate): $m=0.1 . S_{11}(\mathrm{~Pa})$ versus $G_{11}$ (blue); $S_{11}(\mathrm{~Pa})$ versus $G_{22}$ (orange); $l=30 \mathrm{~mm} ; m=0.1$; $n=1$. This graphic shows a softening behavior and a snap-backlike transition due to localized elastic unloading at the right side of the reference domain.

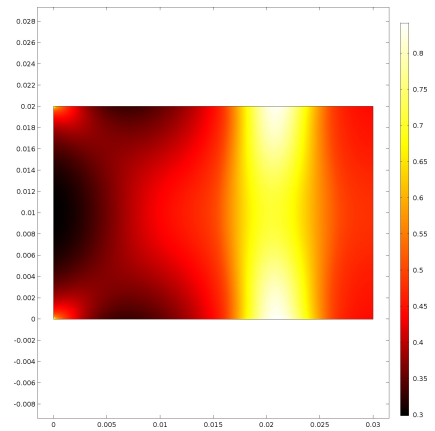

Figure 17. Tensile test (plain plate): $m=0.1$. Color map of $\omega$ at failure point; $l=30 \mathrm{~mm} ; m=0.1 ; n=1$.

relationship. In Figure 16, the stress-strain curve is shown for the test in Figure 6, when $m=0.1$. Clearly, in this case the weight of the second-gradient contribution is sufficiently high to see a decrease of stress as strain increases. It is also relevant that, for such value of $m$, a snap-back like transition, due to elastic unloading of the region of the specimen adjacent to the right side, occurs. Indeed, as shown in Figure 17, failure occurs at a banded region in the middle of the specimen. It is worth remarking that this phenomenon is not a snap-back in the proper sense of the word, because there is no instability involved in the process. In Figure 20 the contour plot of damage at failure point is shown for the same experiment and for a different width of the rectangular specimen, in order to show that the position of the damage band remains unchanged. This evidence is highlighted in Figure 18. In Figure 19 contour plots of the components of the stress tensor are shown. In Figure 21, the contour plot of damage at failure point for the test in Figure 6, 


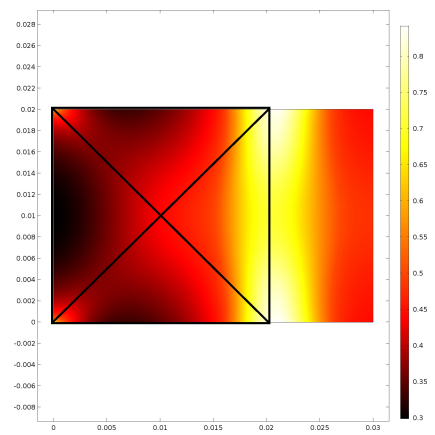

Figure 18. Tensile test (plain plate): $m=0.1$. Color map of $\omega$ at failure point; $l=30 \mathrm{~mm} ; m=0.1 ; n=1$.
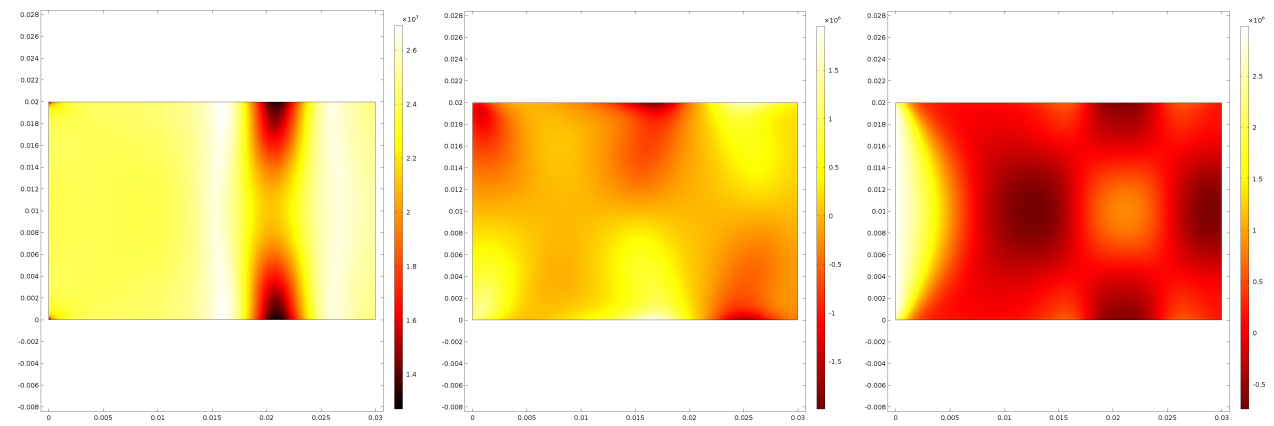

Figure 19. Tensile test (plain plate): $m=0.1$. Color map of $S_{11}(\mathrm{~Pa})$ (left), $S_{12}(\mathrm{~Pa})$ (center), $S_{22}(\mathrm{~Pa})$ (right) at failure point; $l=30 \mathrm{~mm} ; m=0.1 ; n=1$.

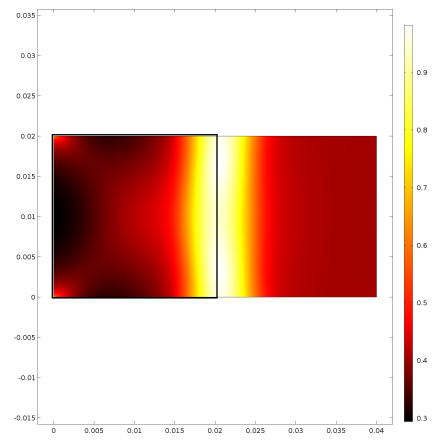

Figure 20. Tensile test (plain plate): $m=0.1$. Color map of $\omega$ at failure point; $l=40 \mathrm{~mm} ; m=0.1 ; n=1$.

when $m=0.1$, is shown for $n=0$, i.e., the second-gradient coefficients (or the microstructure) are not sensitive at all to the internal state of damage. It is clear 


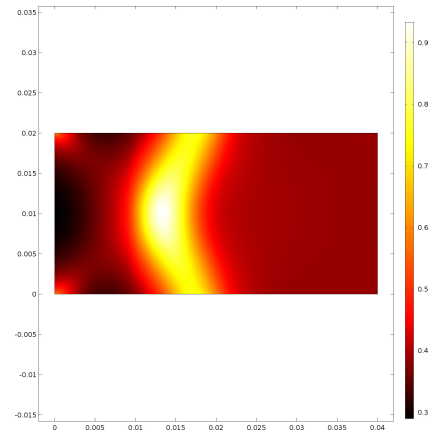

Figure 21. Tensile test (plain plate): $m=0.1$. Color map of $\omega$ at failure point; $l=40 \mathrm{~mm} ; m=0.1 ; n=0$.

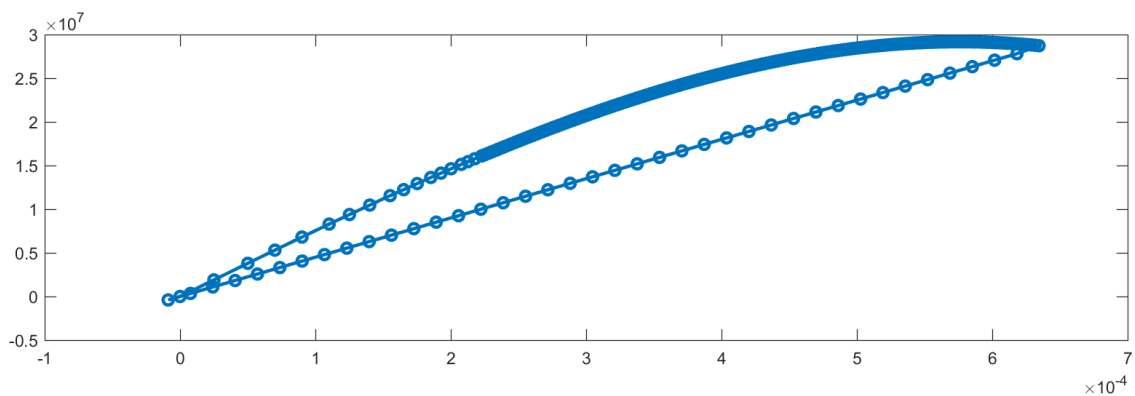

Figure 22. Tensile test (plain plate): $m=0.1 . S_{11}(\mathrm{~Pa})$ versus $G_{11} ; l=30 \mathrm{~mm} ; m=0.1 ; n=1$. This graphic shows dependence of the system upon the path (loading-unloading). The measure of the area inside the cycle is roughly equal to the dissipated energy. After the unloading, the specimen comes back to the initial unstressed configuration. No plastic effect is taken into account.

that the main difference between Figures 17 and 21 is the shape of the damage band. The two figures were obtained under the same conditions, reported in their captions, except for the value of $n$. The damage band is distorted for $n=0$. In Figures 23, 24 , and 25 the stress-strain curve, the damage contour plot at failure point, and the contour plot of the components of the stress tensor for the test described in Figure 6 (with $m=1$ ) are shown, respectively. As is clear from Figure 23, in this case no snap-back-like transition is observed. This is due to the fact that the region of the specimen subject to elastic unloading does not include the point where stress and strain are evaluated in Figure 23, i.e., the middle point of the right side, since, as shown in Figure 24, the damage band touches the right boundary of the specimen. In Figure 22 dependence of the system upon the path is shown in a loading-unloading cycle for the data reported in the caption. Since no plastic effects 


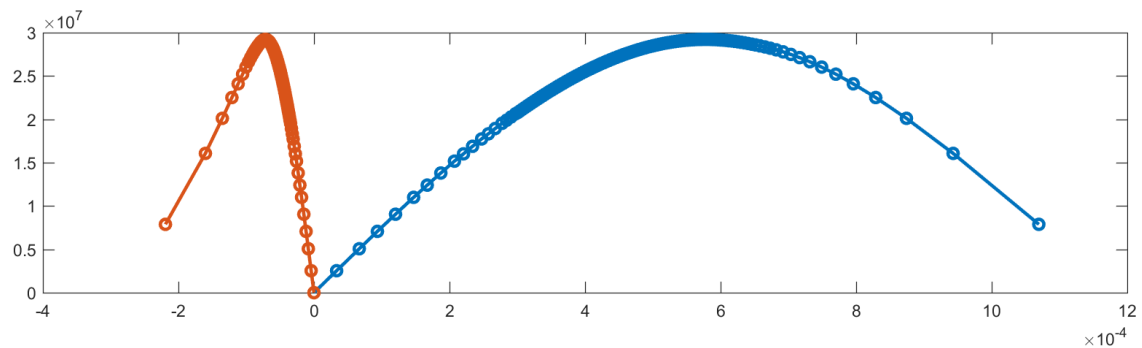

Figure 23. Tensile test (plain plate): $m=1$. $S_{11}(\mathrm{~Pa})$ versus $G_{11}$ (blue); $S_{11}(\mathrm{~Pa})$ versus $G_{22}$ (orange); $l=30 \mathrm{~mm} ; m=1 ; n=1$. This graphic shows strain-gradient-enabled softening.

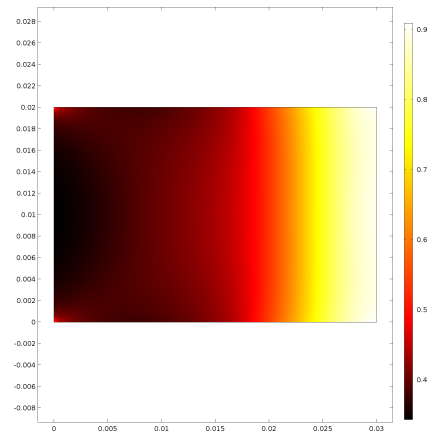

Figure 24. Tensile test (plain plate): $m=1$. Color map of $\omega$ at failure point; $l=30 \mathrm{~mm} ; m=1 ; n=1$.
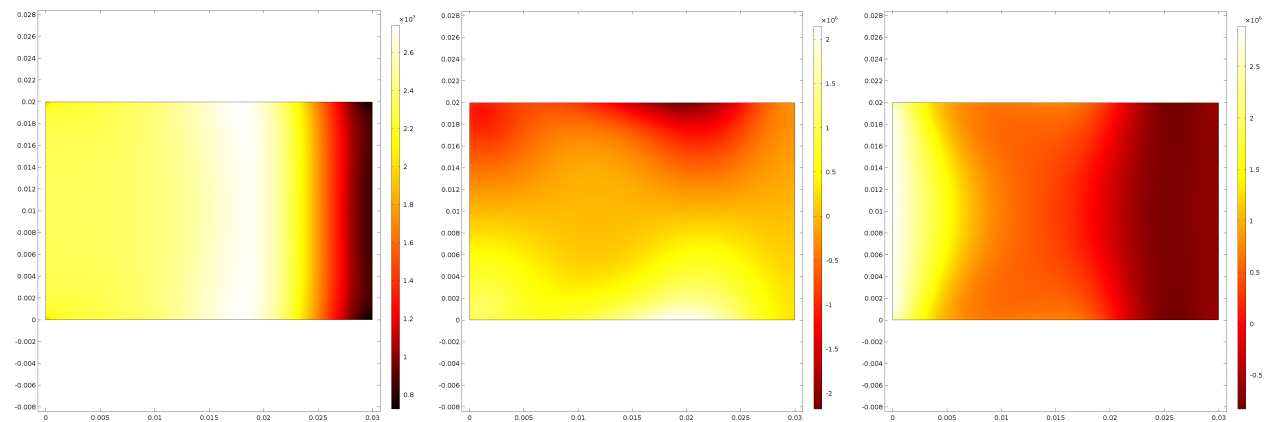

Figure 25. Tensile test (plain plate): $m=1$. Color map of $S_{11}(\mathrm{~Pa})$ (left), $S_{12}(\mathrm{~Pa})$ (center), $S_{22}(\mathrm{~Pa})$ (right) at failure point; $l=30 \mathrm{~mm}$; $m=1 ; n=1$.

are taken into account in the model, after the unloading the specimen comes back to the initial unstressed configuration. Finally, in Figures 26 and 27 mesh-dependency 


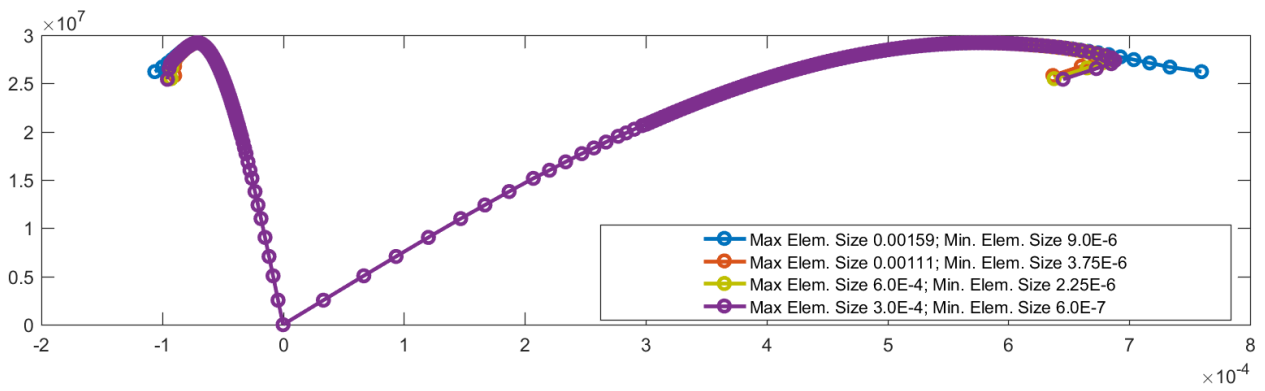

Figure 26. $S_{11}(\mathrm{~Pa})$ versus $G_{11}$ (left); $S_{11}(\mathrm{~Pa})$ versus $G_{22}$ (right); $l=30 \mathrm{~mm} ; m=0.1 ; n=1$. Mesh-dependency study for the tensile test (plain plate). The mesh is triangular and Delaunay-tessellated. Maximum and minimum element sizes are expressed in meters.

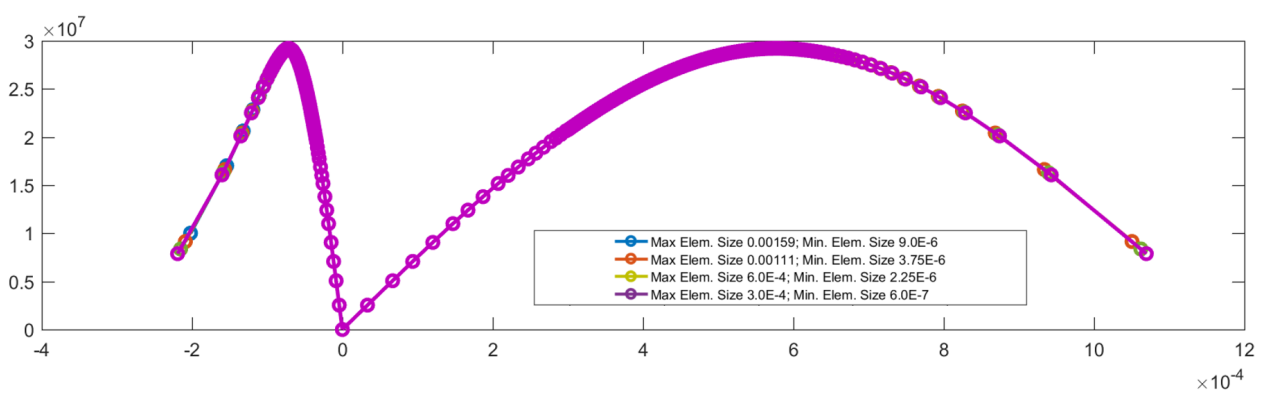

Figure 27. $S_{11}(\mathrm{~Pa})$ versus $G_{11}$ (left); $S_{11}(\mathrm{~Pa})$ versus $G_{22}$ (right); $l=30 \mathrm{~mm} ; m=1 ; n=1$. Mesh-dependency study for the tensile test (plain plate). The mesh is triangular and Delaunay-tessellated. Maximum and minimum element sizes are expressed in meters.

parametric studies, parametrized over the element size, are reported, respectively, for $m=0.1$ and $m=1$, in the case of the test in Figure 6.

The size of the load parameter step $\Delta \bar{u}_{i}$ has been tuned in order to avoid stepdependent simulations. The step size can be higher, still keeping a good accuracy in the numerical solution, when the specimen is in a regime which resembles the elastic one, i.e., when the stress-strain dependence is very close to linear, while damage increase is overestimated to a certain extent when the step size is too large. In that case, the load-parameter step has been decreased accordingly.

\section{Conclusion and outlooks}

Continuum damage mechanics, because of the presence of strain localization, deserves a nonlocal generalization. Advantages and challenges of the incorporation of nonlocal effects, by including either the gradient of damage or the second gradient 
of displacement in the internal strain energy, have been discussed. In this paper we have exploited the case of a damage continuum isotropic two-dimensional strain gradient model. In order to obtain a well posed system of PDEs, BCs, and KKT conditions, a variational approach has been used. Indeed, what is worth remarking is that the variational approach developed in this work allows us to recover not only the relevant governing equations and an incremental damage evolution law, but also boundary conditions which lead to a well posed problem [dell'Isola et al. 2015b; dell'Isola and Placidi 2011; dell'Isola et al. 2015a; Piola 2014]. We have shown that, in the present model, the regularizing effect of the strain gradient terms results, most of the time, in an irreversible softening behavior (i.e., the derivative of stress with respect to strain becomes negative) and, sometimes, even in a kind of snap-back-like transition due to localized elastic unloading. This localized elastic unloading, in the examples that have been shown, clearly arises from the vertically banded damage leading to failure, very likely formed by the propagation and combination of damage from the upper- and lower-left corners. In fact, along this damage band the material is relaxed very much in comparison to other regions of the specimen, i.e., the stiffness, there, is much lower than in other regions. Hence, this region is elongating much more than other regions which, in order to keep the global strain as the one assigned by boundary conditions, are shrunk. We have shown that the position of the damage band depends upon $m$, which weights the second-gradient contribution to the strain energy, and its shape depends upon $n$, which relates such contribution to damage. No snap-back-like transition due to localized elastic unloading at the right side of the reference domain occurs when the damage band touches the right boundary. Simulations were performed also using quadratic Lagrange elements (instead of Hermite cubic elements, for $m>0$ ) and squared meshes (instead of triangular Delaunay-tessellated meshes), confirming the reliability of the results in such a numerically challenging task.

The outlooks of this work are the following. First of all, it would be interesting to exploit the 3D case, in order to understand if geometrical effects due to a higher domain dimensionality could give rise to qualitatively different damage patterns. Then, in order to fit experimental data, it is necessary to develop methods for the experimental and/or theoretical characterization of the constitutive coefficients related to damage. Having a theoretical characterization of such coefficients would be ideal, because it would yield the identification of model parameters related to damage less costly. A promising approach to achieve this goal is to use identification methods based on granular micromechanics [Misra and Singh 2015; 2013], where some kind of damage mechanisms are modeled at the microlevel. Another important line of research that could be potentially pursued starting from this paper would deal with the extension of the present work in order to include plastic phenomena [Contrafatto and Cuomo 2002]. A further numerical campaign has to 
be carried out in order to better investigate the variety of phenomena that can be exhibited by this model. There are a number of research questions which are still open; e.g., does higher $m$ with $0.05<m<0.1$ always imply rightmost damage band? In any case, on the basis of the results shown in this paper, we can formulate several research hypotheses. A better understanding of the simple model studied in this paper could allow significant advancements for the development of a more involved model including, e.g., anisotropy, large deformations, plasticity, different dissipated energy, etc., based upon the same working principle, and that will be the subject of further investigations. We observe that it could be of interest to generalize the results of this paper, e.g., to the modeling of damaging processes in bone tissues [Andreaus et al. 2015; 2014; Giorgio et al. 2016a; 2016b] and of cementitious and granular materials [Misra and Singh 2015; 2013; Yang and Misra 2012; Yang et al. 2011]. Finally, this approach could be especially useful also for the modeling of damage in 2D structures such as pantographic sheets, fiber textile composites, and elastic nets [Spagnuolo et al. 2017; Turco et al. 2017; 2016; dell'Isola et al. 2016; Eremeyev et al. 2017; Battista et al. 2015].

\section{Acknowledgements}

Misra was supported by United States NSF grant CMMI 1727433. This work was also supported by a grant from the Government of the Russian Federation (contract number 14.Y26.31.0031).

\section{References}

[Ambrosio and Tortorelli 1990] L. Ambrosio and V. M. Tortorelli, "Approximation of functionals depending on jumps by elliptic functionals via $\Gamma$-convergence", Comm. Pure Appl. Math. 43:8 (1990), 999-1036.

[Amor et al. 2009] H. Amor, J.-J. Marigo, and C. Maurini, "Regularized formulation of the variational brittle fracture with unilateral contact: numerical experiments", J. Mech. Phys. Solids 57:8 (2009), 1209-1229.

[Andreaus et al. 2014] U. Andreaus, I. Giorgio, and T. Lekszycki, "A 2-D continuum model of a mixture of bone tissue and bio-resorbable material for simulating mass density redistribution under load slowly variable in time", Z. Angew. Math. Mech. 94:12 (2014), 978-1000.

[Andreaus et al. 2015] U. Andreaus, I. Giorgio, and A. Madeo, "Modeling of the interaction between bone tissue and resorbable biomaterial as linear elastic materials with voids", Z. Angew. Math. Phys. 66:1 (2015), 209-237.

[Battista et al. 2015] A. Battista, C. Cardillo, D. Del Vescovo, N. L. Rizzi, and E. Turco, "Frequency shifts induced by large deformations in planar pantographic continua", Nanomech. Sci. Tech. 6:2 (2015), 161-178.

[Bažant 1980] Z. P. Bažant, "Work inequalities for plastic fracturing materials", Int. J. Solids Struct. 16:10 (1980), 873-901.

[Bažant and Jirásek 2002] Z. P. Bažant and M. Jirásek, "Nonlocal integral formulations of plasticity and damage: survey of progress", J. Eng. Mech. 128:11 (2002), 1119-1149. 
[Bažant and Pijaudier-Cabot 1988] Z. P. Bažant and G. Pijaudier-Cabot, "Nonlocal continuum damage, localization instability and convergence”, J. Appl. Mech. 55:2 (1988), 287-293.

[Bourdin et al. 2008] B. Bourdin, G. A. Francfort, and J.-J. Marigo, "The variational approach to fracture", J. Elasticity 91:1-3 (2008), 5-148.

[Comi 1999] C. Comi, "Computational modelling of gradient-enhanced damage in quasi-brittle materials", Mech. Cohesive-Frictional Mat. 4:1 (1999), 17-36.

[Contrafatto and Cuomo 2002] L. Contrafatto and M. Cuomo, "A new thermodynamically consistent continuum model for hardening plasticity coupled with damage”, Int. J. Solids Struct. 39:25 (2002), 6241-6271.

[dell'Isola and Placidi 2011] F. dell' Isola and L. Placidi, "Variational principles are a powerful tool also for formulating field theories", pp. 1-15 in Variational models and methods in solid and fluid mechanics, edited by F. dell'Isola and S. Gavrilyuk, CISM Courses and Lect. 535, Springer, 2011.

[dell'Isola et al. 2015a] F. dell'Isola, U. Andreaus, and L. Placidi, "At the origins and in the vanguard of peridynamics, non-local and higher-gradient continuum mechanics: an underestimated and still topical contribution of Gabrio Piola”, Math. Mech. Solids 20:8 (2015), 887-928.

[dell' Isola et al. 2015b] F. dell'Isola, P. Seppecher, and A. Della Corte, "The postulations à la d'Alembert and à la Cauchy for higher gradient continuum theories are equivalent: a review of existing results", Proc. A. 471:2183 (2015), art. id. 20150415.

[dell' Isola et al. 2016] F. dell'Isola, I. Giorgio, M. Pawlikowski, and N. L. Rizzi, "Large deformations of planar extensible beams and pantographic lattices: heuristic homogenization, experimental and numerical examples of equilibrium", Proc. A 472:2185 (2016), art. id. 20150790.

[Eremeyev et al. 2017] V. A. Eremeyev, F. dell'Isola, C. Boutin, and D. Steigmann, "Linear pantographic sheets: existence and uniqueness of weak solutions", J. Elasticity (online publication November 2017).

[Forest 2009] S. Forest, "Micromorphic approach for gradient elasticity, viscoplasticity, and damage", J. Eng. Mech. 135:3 (2009), 117-131.

[Giorgio et al. 2016a] I. Giorgio, U. Andreaus, and A. Madeo, "The influence of different loads on the remodeling process of a bone and bioresorbable material mixture with voids", Contin. Mech. Thermodyn. 28:1-2 (2016), 21-40.

[Giorgio et al. 2016b] I. Giorgio, U. Andreaus, D. Scerrato, and F. dell'Isola, "A visco-poroelastic model of functional adaptation in bones reconstructed with bio-resorbable materials", Biomech. Model. Mechanobiol. 15:5 (2016), 1325-1343.

[Hill 1948] R. Hill, "A variational principle of maximum plastic work in classical plasticity", Quart. J. Mech. Appl. Math. 1:1 (1948), 18-28.

[Lorentz and Andrieux 2003] E. Lorentz and S. Andrieux, "Analysis of non-local models through energetic formulations", Int. J. Solids Struct. 40:12 (2003), 2905-2936.

[Maier 1970] G. Maier, "A minimum principle for incremental elastoplasticity with non-associated flow laws”, J. Mech. Phys. Solids 18:5 (1970), 319-330.

[Marigo 1989] J. J. Marigo, "Constitutive relations in plasticity, damage and fracture mechanics based on a work property", Nuclear Eng. Des. 114:3 (1989), 249-272.

[Miehe et al. 2016] C. Miehe, F. Aldakheel, and A. Raina, "Phase field modeling of ductile fracture at finite strains: a variational gradient-extended plasticity-damage theory", Int. J. Plasticity 84 (2016), 1-32.

[Misra and Singh 2013] A. Misra and V. Singh, "Micromechanical model for viscoelastic materials undergoing damage", Contin. Mech. Thermodyn. 25:2-4 (2013), 343-358. 
[Misra and Singh 2015] A. Misra and V. Singh, "Thermomechanics-based nonlinear rate-dependent coupled damage-plasticity granular micromechanics model”, Contin. Mech. Thermodyn. 27:4-5 (2015), 787-817.

[Mühlich et al. 2013] U. Mühlich, L. Zybell, G. Hütter, and M. Kuna, "A first-order strain gradient damage model for simulating quasi-brittle failure in porous elastic solids", Arch. Appl. Mech. 83:6 (2013), 955-967.

[Oliver-Leblond et al. 2016] C. Oliver-Leblond, H. Dumontet, and D. Kondo, "A micro-mechanics based strain gradient damage model: formulation and solution for the torsion of a cylindrical bar", Eur. J. Mech. A Solids 56 (2016), 19-30.

[Peerlings et al. 2001] R. H. J. Peerlings, M. G. D. Geers, R. de Borst, and W. A. M. Brekelmans, "A critical comparison of nonlocal and gradient-enhanced softening continua", Int. J. Solids Struct. 38:44-45 (2001), 7723-7746.

[Pham and Marigo 2010a] K. Pham and J.-J. Marigo, “Approche variationnelle de l'endommagement, I: Les concepts fondamentaux", C. R. Mécanique 338:4 (2010), 191-198.

[Pham and Marigo 2010b] K. Pham and J.-J. Marigo, "Approche variationnelle de l'endommagement, II: Les modèles à gradient”, C. R. Mécanique 338:4 (2010), 199-206.

[Pham et al. 2011a] K. Pham, H. Amor, J.-J. Marigo, and C. Maurini, "Gradient damage models and their use to approximate brittle fracture", Int. J. Damage Mech. 20:4 (2011), 618-652.

[Pham et al. 2011b] K. Pham, J.-J. Marigo, and C. Maurini, "The issues of the uniqueness and the stability of the homogeneous response in uniaxial tests with gradient damage models", J. Mech. Phys. Solids 59:6 (2011), 1163-1190.

[Piola 2014] G. Piola, The complete works, I, edited by F. dell'Isola et al., Advanced Structured Materials 38, Springer, 2014.

[Placidi 2015] L. Placidi, "A variational approach for a nonlinear 1-dimensional second gradient continuum damage model”, Contin. Mech. Thermodyn. 27:4-5 (2015), 623-638.

[Placidi 2016] L. Placidi, "A variational approach for a nonlinear one-dimensional damage-elastoplastic second-gradient continuum model”, Contin. Mech. Thermodyn. 28:1-2 (2016), 119-137.

[Placidi and El Dhaba 2017] L. Placidi and A. R. El Dhaba, "Semi-inverse method à la Saint-Venant for two-dimensional linear isotropic homogeneous second-gradient elasticity", Math. Mech. Solids 22:5 (2017), 919-937.

[Placidi et al. 2015] L. Placidi, U. Andreaus, A. Della Corte, and T. Lekszycki, "Gedanken experiments for the determination of two-dimensional linear second gradient elasticity coefficients", $Z$. Angew. Math. Phys. 66:6 (2015), 3699-3725.

[Placidi et al. 2017] L. Placidi, U. Andreaus, and I. Giorgio, "Identification of two-dimensional pantographic structure via a linear D4 orthotropic second gradient elastic model", J. Eng. Math. 103 (2017), 1-21.

[Rahali et al. 2016] Y. Rahali, I. Goda, and J. F. Ganghoffer, "Numerical identification of classical and nonclassical moduli of 3D woven textiles and analysis of scale effects", Compos. Struct. 135 (2016), 122-139.

[Reddy 2011a] B. D. Reddy, "The role of dissipation and defect energy in variational formulations of problems in strain-gradient plasticity, I: Polycrystalline plasticity", Contin. Mech. Thermodyn. 23:6 (2011), 527-549. Erratum in 24:1 (2012), 79.

[Reddy 2011b] B. D. Reddy, "The role of dissipation and defect energy in variational formulations of problems in strain-gradient plasticity, II: Single-crystal plasticity", Contin. Mech. Thermodyn. 23:6 (2011), 551-572. 
[Sicsic and Marigo 2013] P. Sicsic and J.-J. Marigo, "From gradient damage laws to Griffith's theory of crack propagation", J. Elasticity 113:1 (2013), 55-74.

[Spagnuolo et al. 2017] M. Spagnuolo, K. Barcz, A. Pfaff, F. dell'Isola, and P. Franciosi, "Qualitative pivot damage analysis in aluminum printed pantographic sheets: numerics and experiments", Mech. Res. Comm. 83 (2017), 47-52.

[Turco et al. 2016] E. Turco, F. dell'Isola, N. L. Rissi, R. Grygoruk, W. H. Müller, and C. Liebold, "Fiber rupture in sheared planar pantographic sheets: numerical and experimental evidence", Mech. Res. Comm. 76 (2016), 86-90.

[Turco et al. 2017] E. Turco, M. Golaszewski, I. Giorgio, and F. D’Annibale, "Pantographic lattices with non-orthogonal fibres: experiments and their numerical simulations", Comp. B 118 (2017), $1-14$.

[Yang and Misra 2012] Y. Yang and A. Misra, "Micromechanics based second gradient continuum theory for shear band modeling in cohesive granular materials following damage elasticity", Int. J. Solids Struct. 49:18 (2012), 2500-2514.

[Yang et al. 2011] Y. Yang, W. Y. Ching, and A. Misra, "Higher-order continuum theory applied to fracture simulation of nanoscale intergranular glassy film", J. Nanomech. Micromech. 1:2 (2011), $60-71$.

[Zybell et al. 2009] L. Zybell, U. Mühlich, and M. Kuna, "Constitutive equations for porous planestrain gradient elasticity obtained by homogenization”, Arch. Appl. Mech. 79:4 (2009), 359-375.

Received 4 Oct 2017. Accepted 26 Apr 2018.

LUCA PLACIDI: luca.placidi@uninettunouniversity.net

Facoltà di Ingegneria, Università Telematica Internazionale Uninettuno, Roma, Italy

EMILIO BARCHIESI: barchiesiemilio@gmail.com

Dipartimento di Ingegneria Strutturale e Geotecnica, Università degli Studi di Roma "La Sapienza", Roma, Italy

ANIL MisRa: amisra@ku.edu

Civil, Environmental and Architectural Engineering Department, University of Kansas, Lawrence, KS, United States 